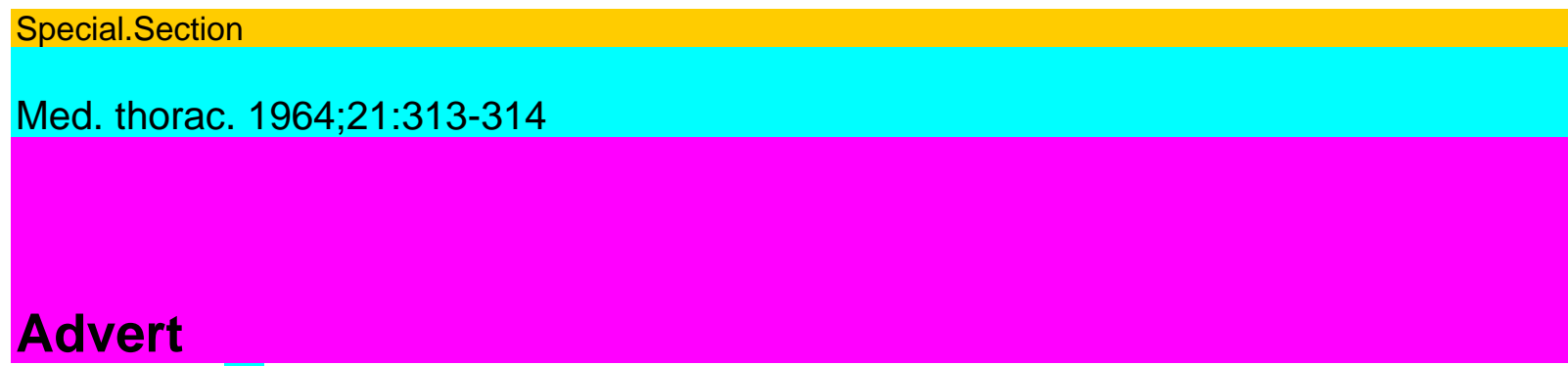

\title{
Competition 1965
}

The Dr. Heinz Karger Memorial Foundation invites the submission of original papers on the following subject:

\section{MICROANGIOLOGICAL PROBLEMS IN ARTERIOSCLEROSIS}

to compete for an award of sFr. 3000.-- The Council of the Foundation will judge the papers and confer the prize.

\section{Conditions}

Language: English, German or French Length of Manuscript: The manuscript shall not exceed 20 typewritten pages, including illustrations, tables and bibliography. The manuscript must be typewritten on one side only, double-spaced; it is to be submitted in duplicate and in accordance with the instructions contained in the "Rules for the Preparation of Manuscripts and Bibliographies". The booklet can be obtained from the publishers free of charge if the request is marked "Competition". Submission date: Manuscripts marked "Competition" must reach the publishers, S. Karger AG, Arnold-Böcklin-Strasse 25, 4000 Basel 11

(Switzerland), not later than August 31, 1965.

Publication: The winning paper will be published in 1965 in one of the

Karger journals.

Preisausschreiben 1965

Die Dr. Heinz Karger-Gedächtnis-Stiftung schreibt einen Preis aus für eine Originalarbeit über das Thema

\section{MIKROANGIOLOGISCHE PROBLEME DER ARTERIOSKLEROSE}

Die Höhe des Preises beträgt sFr. 3000.-. Über die Zuerkennung des Preises entscheidet der

Stiftungsrat.

Bedíngungen

Sprache: Englisch, Deutsch oder Französisch.

Umfang des Manuskriptes: Es soil 20 Schreibmaschinenseiten ein-

schließlich Abbildungen, Tabellen und Literaturverzeichnis nicht

Med. thorac, Vol. 21, No. 6 (1964)

23

überschreiten. Das Manuskript soil einseitig mit doppeltem Zeilenab-stand geschrieben sein, im Doppel eingereicht werden und gemäß un-seren Richtlinien zur Abfassung von Manuskript und Literaturver-zeichnis zusammengestellt und abgefaßt sein (die Broschüre «Das Manuskript* kann beim Verlag kostenlos bezogen werden, wenn die

Anfrage den Vermerk trägt «für Preisausschreiben»). Termín: Das Manuskript ist bis zum 31. August 1965 mit dem Vermerk

«Preisausschreiben» an den Verlag S. Karger AG, Arnold-Böcklin-

Straße 25, 4000 Basel 11 (Schweiz), einzusenden.

Veröffentlíchung: Die mit dem Preis ausgezeichnete Arbeit erscheint im Jahre 1965 in einer der Zeitschriften des Verlages Karger.

\section{Concours 1965}

La Fondation Commemorative Dr. Heinz Karger récompensera d'un prix en espèces, le meilleur travail sur le theme suivant: 


\section{PROBLÈMES MICROANGIOLOGIQUES DE L’ARTÉRIOSCLÉROSE}

Ce prix est fixe à fr.s. 3000.-. Le Conseil de Fondation se prononcera sur le choix du travail et de Cattribution du prix.

Conditions Langue: Anglais, allemand ou français. Volume du manuscrit: Ce dernier ne devra pas dépasser 20 pages dacty-lographiées, figures, tableaux et listes de references y compris. Le manuscrit est à écrire en double exemplaire et sur un côté seulement de chaque feuille; 1'intervalle employe entre les lignes doit être double. Le manuscrit devra être rédigé conformément à nos «Directives sur la Redaction d'un Manuscrit et ГEtablissement d'une Bibliographie» (la brochure peut être obtenue gratuitement auprès de la Maison d'éditions, à condition que la demande porte la mention «pour le concours»). Délaí: Les travaux seront à remettre à la Maison d'éditions, S. Karger S.A., Arnold-Böcklin-Strasse 25, à 4000 Bale 11 (Suisse), jusqu'au 31 aoùt 1965 et devront porter la remarque suivante: «pour le concours ». Publication: Le travail recompense sera publié en 1965, dans une des revues de la Maison d'éditions Karger. 